\title{
MicroRNA-101 inhibits proliferation of pulmonary microvascular endothelial cells in a rat model of hepatopulmonary syndrome by targeting the JAK2/STAT3 signaling pathway
}

\author{
LIGUO WANG $^{1 *}$, LIWEI ZHUANG ${ }^{2 *}$, HAIFANG RONG $^{3}$, YUENING GUO $^{1}$, \\ XIAOHUA LING ${ }^{2}$, RUIFENG WANG ${ }^{2}, \mathrm{XIN}_{\mathrm{YU}^{2}}$ and WEI ZHANG ${ }^{1}$ \\ ${ }^{1}$ Department of Respiratory, First Affiliated Hospital of Harbin Medical University; \\ ${ }^{2}$ Department of Gastroenterology, The Fourth Affiliated Hospital of Harbin Medical University; \\ ${ }^{3}$ Department of Respiratory, The First Hospital of Harbin, Harbin, Heilongjiang 150000, P.R. China
}

Received September 9, 2014; Accepted July 7, 2015

DOI: $10.3892 / \mathrm{mmr} .2015 .4471$

\begin{abstract}
Hepatopulmonary syndrome (HPS) is one of the reasons for the mortality of patients with perioperative liver disease. Intrapulmonary vascular dilatation is the most important mechanism underlying HPS, and it primarily occurs due to cell proliferation. Inhibiting the proliferation of pulmonary microvascular endothelial cells (PMVECs) may provide a novel strategy to prevent HPS. MicroRNAs (miRNAs) regulate gene expression and are crucial in cell proliferation. To investigate the mechanism underlying the proliferation of PMVECs in HPS, PMVECs were isolated from rat models of HPS. It was demonstrated that interleukin (IL)- 6 could stimulate the janus kinase 2(JAK2)/ signal transducer and activator of transcription (STAT3) signaling pathway, which promotes the cell proliferation of PMVECs. JAK2 is a novel target gene of miR-101 and it was shown that miR-101 could inhibit cell proliferation by targeting the IL-6/JAK2/STAT3 signal pathway. In conclusion, the present study demonstrated that miR-101 could inhibit the proliferation of PMVECs by targeting the IL-6/JAK2/STAT3 signaling pathway. This clarifies the role of miR-101 in HPS and provides the theoretical basis of the pathogenesis of HPS.
\end{abstract}

\section{Introduction}

Hepatopulmonary syndrome (HPS) is defined as the presence of the triad of an arterial oxygenation defect, intrapulmonary vasodilation, and the presence of liver disease $(1,2)$. It usually occurs in patients diagnosed with cirrhosis and is

Correspondence to: Dr Wei Zhang, Department of Respiratory, First Affiliated Hospital of Harbin Medical University, 23 Youzheng Street, Harbin, Heilongjiang 150000, P.R. China E-mail: zhang_wei18@126.com

Contributed equally

Key words: microRNA-101, janus kinase 2, signal transducer and activator of transcription, hepatopulmonary syndrome, interleukin-6 characterized by a lack of arterial oxygen $(1,2)$. However, HPS is under-diagnosed in patients with late-stage liver disease as there is no consensus on the diagnostic criteria. Liver transplantation is hypothesized to be a relatively effective treatment for HPS (3-5). The molecular mechanism underlying HPS is reported to be associated with numerous factors such as lipopolysaccharide, nitric oxide and tumor necrosis factor (TNF)- $\alpha$ among others (6-11); however it remains unclear. Thus, further research is required.

MicroRNAs (miRNAs) are small noncoding RNAs 22-nucleotides in length, which function as important regulators in gene expression $(12,13)$. miRNAs inhibit the expression of target genes by specifically binding and cleaving mRNAs or inhibiting their translation. There are 2,578 miRNAs that are involved in various cellular physiological and pathological processes $(12,13)$. However, to date, there are no reports on the role of miRNAs in HPS.

To investigate the involvement of miRNAs in the regulation of pulmonary microvascular endothelial cell (PMVEC) proliferation, an HPS model was induced and PMVECs were isolated. This study aimed to identify the molecular mechanism of the effect of miR-101 on pulmonary microvascular endothelial cells in the HPS rat model.

\section{Materials and methods}

Reagents and antibodies. Rabbit anti-human phospho-janus kinase(Jak)1 polyconal antibody (cat.no.3331),rabbit anti-human Jak1 polyconal antibody (cat. no. 3332), phospho-Jak2 rabbit mAb (cat. no. 3376), JAK2 polyconal antibody (cat. no. 3773), phospho-Jak3 rabbit mAb (cat. no. 5031), Jak3 polyconal antibody (cat. no. 3775), phospho-Stat3 rabbit mAb (cat. no. 9154) and Stat 3 mouse mAb (cat. no. 9139) were all purchased from Cell Signaling Technology, Inc. (Danvers, MA, USA). Each antibody was used at a dilution of 1:1,000. Pim-1 mAb (cat. no. sc-374116) and Mcl-1mAb (cat. no. sc-377487) were purchased from Santa Cruz Biotechnology Inc. (Santa Cruz, CA, USA) and the dilution was 1:500. Primary GAPDH mAb and secondary antibodies conjugated to horseradish peroxidase $(1: 5,000)$ were ordered from Kang-Chen Biotech (Shanghai, China). 
HPS rat model. HPS rat models were constructed according to the protocols of the National Institutes of Health. The procedure of the study was approved by the committee on Animal Research of First Affiliated Hospital of Harbin Medical University. A total of 20 male Sprague Dawley rats (weight, 180-220 g; age, 12 weeks) were used in this study. The HPS rat models were successfully constructed in our study. At $\mathrm{PaO}_{2}$ $<85 \mathrm{~mm} \mathrm{Hg}$, the blood serum of rats with $\mathrm{P}(\mathrm{A}-\mathrm{a}) \mathrm{O}_{2}>18 \mathrm{~mm}$ $\mathrm{Hg}$ and pulmonary vasodilatation (PVD) found on pathological sections was used for this study. Serum was separated from CBDL blood samples (centrifugation at 2,000 $\mathrm{x} \mathrm{g} / \mathrm{min}$ for $10 \mathrm{~min}$ at $4^{\circ} \mathrm{C}$ ) within $20 \mathrm{~min}$ of collection, was carefully handled to avoid hemolysis, and was stored at $-80^{\circ} \mathrm{C}$.

PMVEC isolation and culture. PMVECs were isolated from the rat lung as described previously (11). Briefly, rat peripheral tissue (2-5 g) was extracted from normal Sprague Dawley rats. The tissue was washed using phosphate-buffered saline, cut into sections, digested in $0.3 \%$ type II collagenase (Sigma-Aldrich Canada Co., Oakville, ON, Canada) for $45 \mathrm{~min}$ at $37^{\circ} \mathrm{C}$, filtered through $100-\mu \mathrm{m}$ mesh and centrifuged at $500 \mathrm{x}$ g. After washing with PBS, the cell pellet was resuspended in binding buffer and incubated for $20 \mathrm{~min}$ at $4^{\circ} \mathrm{C}$ with magnetic microbeads (Dynal Inc., Lake Success, NY, USA) coated with anti-CD31 antibody. The microbeads were used to recruit cells, which were then washed five times using PBS, resuspended in 10\% Endothelial Growth Medium-2 (EGM-2, Cambrex Bio Science Inc., Walkersville, $\mathrm{MD}, \mathrm{USA}$ ) and incubated for $8-12 \mathrm{~h}$ at $37^{\circ} \mathrm{C}$ in $5 \% \mathrm{CO}_{2} / 21 \%$ $\mathrm{O}_{2}$. The growth medium was changed to fresh medium on the third day of culture in order to remove unattached cells. The fibroblasts were removed 7-10 days after seeding the cells to make PMVEC clusters grow. PMVECs were purified again with anti-CD31-coated magnetic microbeads. PMVECs were seeded in a $25-\mathrm{cm}^{2}$ flask, passaged at a ratio of 1:3 and used for experiments at passage 2-3.

Lentiviral vector construction and transduction. Mature miR-101 antagomir oligonucleotide was synthesized, amplified and cloned into GV232-Puro Vectors by Genechem Co., Ltd. (Shanghai, China). The nucleotides of the insertions were confirmed by DNA sequencing. Lentivirus packaging was performed in $293 \mathrm{~T}$ cells using Lipofectamine 2000 (Invitrogen Life Technologies, Carlsbad, CA, USA) according to the manufacturer's instructions. When analyzing cellular function, cells were transduced with the lentivirus and selected with puromycin. miR-101 expression was verified by reverse transcription-quantitative polymerase chain reaction (RT-qPCR).

Small interfering (si)RNA transfection. Transfection was performed according to the manufacturer's instructions using Lipofectamine 2000 reagent. JAK2 and STAT3 siRNAs were ordered from Santa Cruz Biotechnology Inc. Briefly, 2x10 PMVECs were seeded in 6-well plates. The following day, the cells were transfected with JAK2 or STAT3 siRNA (100 nM) and growth medium was changed after $5 \mathrm{~h}$. JAK2 and STAT3 mRNA and protein levels were examined by RT-qPCR and western blotting, respectively. The transfected cells were used for various assays. For reporter assays, the pGL3-basic vector was co-transfected with siRNAs and luciferase assays were performed $48 \mathrm{~h}$ after transfection.

miRNA target prediction dual luciferase assay. Cells were seeded on 24-well plates and transfected with plasmids carrying the $3^{\prime}$ untranslated region (UTR) of JAK2 and miR-101 using Lipofectamine 2000 according to the manufacturer's instructions. After transfection $(6 \mathrm{~h})$, the medium was changed, and the cells were incubated at $37^{\circ} \mathrm{C}$ for 2 days. Cells were washed once with phosphate-buffered saline and luciferase assays were performed using the Dual Luciferase Assay system (Promega Corporation, Madison, WI, USA) according to the manufacturer's instructions. Readings were taken with a Lumat LB 9507 (Berthold Technologies GmbH, Bad Wildbad, Germany). miR-101 target genes were predicted based on the online bioinformatics software, including TargetScan (http:// www.targetscan.org/vert_61/) and miRbase (http://www. mirbase.org).

$R T$ - $q P C R$. Total RNA was extracted from the PMVECs transfected with miRNA or siRNA, or treated with interleukin (IL)-6 at indicated time points using the Tripure Isolation Reagent. Firstly, total RNA $(2 \mu \mathrm{g})$ was reverse transcribed into cDNA in the reaction system with Oligo (dT)15 (Sangon Biotech Co. Ltd., Shanghai, China), dNTP (Sangon Biotech Co. Ltd.), and the reaction buffer supplied with the M-MLV reverse transcriptase (Promega Corporation). qPCR was performed using Fast SYBR Green master mix (Life technologies, Grand Island, NY, USA). Primer sequences were as follows: TNF- $\alpha$, forward 5'-cgacgtggaactggcagaag-3' and reverse 5'-ccgagaactgccgtctc-3'; IL-1, forward 5'-gecttgaaggta ctgtatctgcaca-3' and reverse 5'-cgaaggccactccacgtctc-3'; IL-6, forward 5'-gatgctggtgacaaccacg-3' and reverse 5'-gacttcctgaga ccgaaaca-3'; IL-8, forward 5'-taggcatcttcgtccgtccctgt-3' and reverse 5'-agagggttactttctata-3'; miR-101, forward 5'-acactccag ctgggtacagtactgtgat-3' and reverse 5'-ctcaactggtgtcgtggagtcggc aattcagttgagtattgact-3'; JAK1, forward 5'-agctgtgcatcagggecg ccc-3' and reverse 5'-cgccgggactacgtgtcga-3'; JAK2, forward 5'-caccaacattacagaggcataata-3' and reverse 5'-gaacgacgaagc ttctttctgag-3'; JAK3, forward 5'-accgagaccttccgtgtgggg-3' and reverse 5'-aggcgaaggtcctacaccggcag-3'; STAT3, forward 5'-cac acgctacctggagcagctg-3' and reverse 5'-actatctcctgtaacctgag-3'; GAPDH, forward 5'-tgtgaaccacggagagggt-3' and reverse 5'-ggc atggactgtggtcatga-3'; snRNA, forward 5'-ctcgcttcggcagcaca-3' and reverse 5'-aacgcttcacgaatttgcgt-3'. TaqMan miRNA assays were purchased from Life technologies and used to quantify mature miRNAs following the manufacturer's instructions. U6 snRNA was used as an internal control. GAPDH and U6 RNA was used the initial control for RNA and miRNA respectively. Specific primer were ordered from Invitrogen Life Technologies.

Western blotting. Total protein was extracted from the cells and its concentration was determined using a Bradford assay (Bio-Rad, Philadelphia, PA, USA). Protein was separated by SDS-PAGE and transferred to membranes (Millipore, Bedford, MA, USA) at $80 \mathrm{~V}$ for $2 \mathrm{~h}$ at $4^{\circ} \mathrm{C}$. The membranes were blocked in 5\% non-fat dry milk in PBST then incubated with primary antibodies in PBS, washed with PBST and then incubated with secondary antibodies conjugated to 

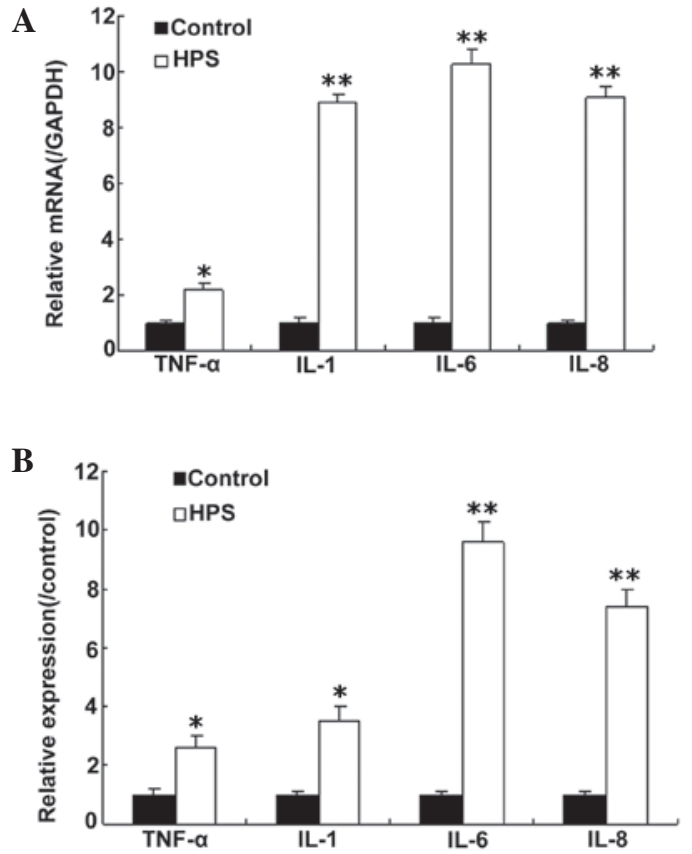

Figure 1. IL-6 is induced in rat models of HPS. (A) TNF- $\alpha$, IL-8, IL-1 and IL-6 mRNA expression was confirmed by reverse transcription-quantitative polymerase chain reaction. (B) IL-6 protein is increased in lung tissue of HPS models as observed using ELISA. ${ }^{*} \mathrm{P}<0.05,{ }^{* *} \mathrm{P}<0.01$ vs. Control. IL, interleukin; HPS hepatopulmonary syndrome; TNF, tumor necrosis factor.

A

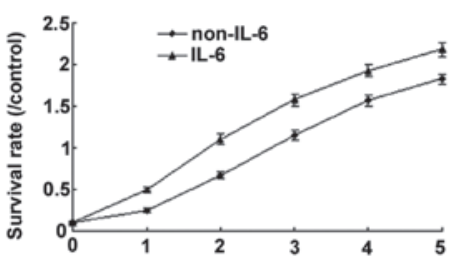

B
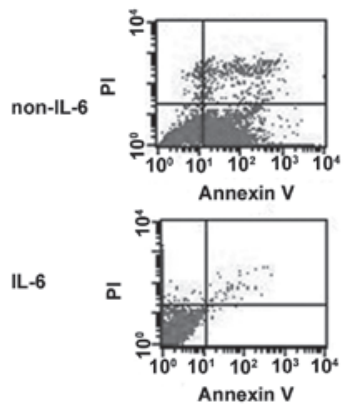

C

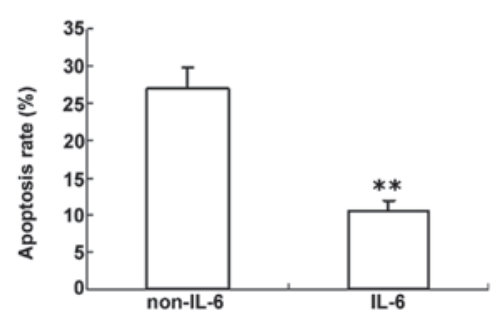

Figure 2. IL-6 promotes in vitro cell proliferation and protects against apoptosis of PMVECs. (A) PMVECs were cultured in 96-well plates at $2 \times 10^{3}$ cells per well for 1,3,5 or 7 days. Cell survival was assessed by an MTT assay as described above. Data are presented as the mean \pm standard deviation of two separate experiments with six samples. (B) PMVECs were cultured in 6-well plates at $2 \times 10^{5}$ cells per well and LPS was added for $12 \mathrm{~h}$. The cell apoptosis was assessed by flow cytometry. (C) Quantification of apoptosis rate. ${ }^{* *} \mathrm{P}<0.01$ vs. non-IL-6. IL, interleukin; PMVECs, pulmonary microvascular endothelial cells; LPS, lipopolysaccharide.

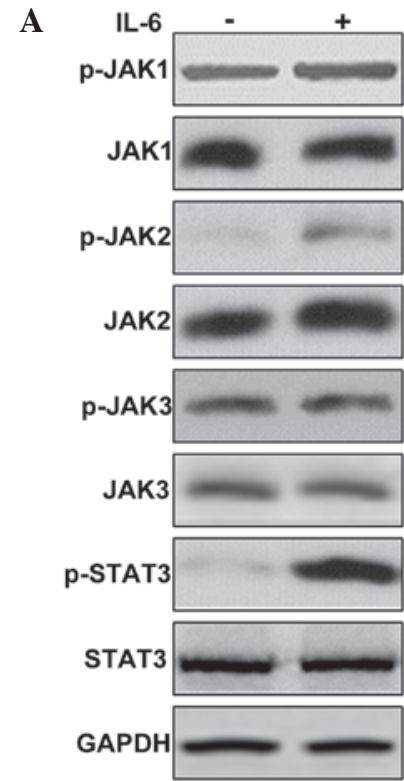

B

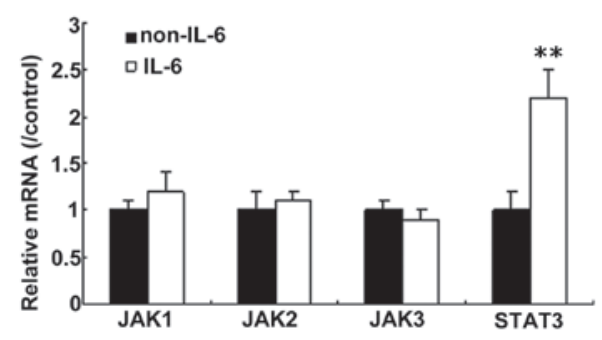

Figure 3. IL-6 activates the JAK/STAT3 signaling pathway in PMVECs (A) IL-6 upregulated JAK and STAT3 expression in PMVECs. Total protein was isolated from the cells, and p-JAK, JAK1, 2 and 3, p-STAT3, STAT3, and GAPDH protein were detected by western blotting. (B) IL-6 was not observed to effect the levels of JAK and STAT3 mRNA in PMVECs. ${ }^{* *} \mathrm{P}<0.01$ vs. non-IL-6. Total RNA was isolated from the cells, and JAK and STAT3 mRNA was detected by reverse transcription-quantitative polymerase chain reaction. The results are representative of three independent experiments with similar results. IL, interleukin; JAK, janus kinase; STAT, signal transducer and activator of transcription; p-, phosphorylated; PMVECs, pulmonary microvascular endothelial cells.

horseradish peroxidase in PBST for $1 \mathrm{~h}$ at room temperature. Membranes were washed again in PBST three times and bands were visualized on X-ray films using an enhanced chemiluminescence detection system.

MTT assay. Cells $\left(10^{3}-10^{4}\right)$ were transfected with miR-101, miR-control or STAT3 siRNA, or treated with IL-6 in $6-\mathrm{cm}$ plates, and added into each well of a 96-well plate after 24-h transfection. The cells were cultured for 1,2, 3, 4 and 5 days. MTT solution (20 $\mu \mathrm{l}$ of $5 \mathrm{mg} / \mathrm{ml})$ was added, and the plate was incubated for $4 \mathrm{~h}$ at $37^{\circ} \mathrm{C}$. The supernatant was discarded and $150 \mu \mathrm{l}$ dimethyl sulfoxide was added to each well until crystals dissolved completely. The absorbance value of each well was measured using an ELISA reader with $490 \mathrm{~nm}$ as the test wavelength and $280 \mathrm{~nm}$ as the reference wavelength.

Apoptosis assay. Cells $\left(2 \times 10^{5}\right)$ were seeded in 6-well plates, cultured and then harvested. The cells were washed with PBS and then stained with $5 \mu \mathrm{l}$ Annexin V and $5 \mu \mathrm{l}$ propidium iodide (PI) for $15 \mathrm{~min}$ at room temperature in the dark 
A

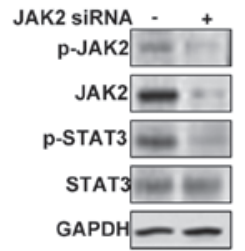

B 2.5 . $\cdots \cdot \cdots$ control siRnA

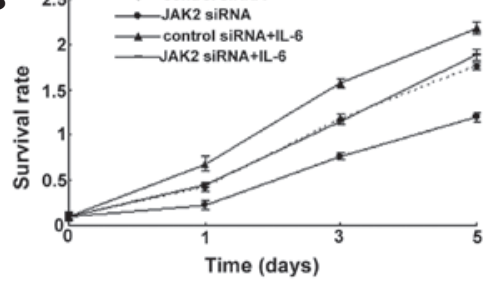

C

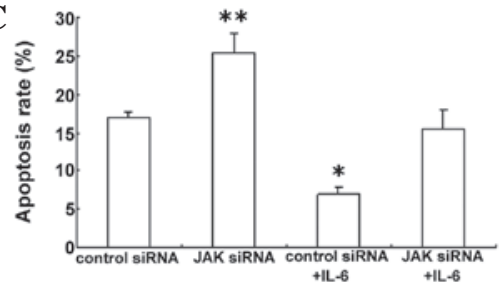

D

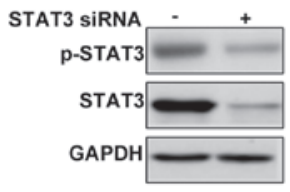

$\mathbf{E}$

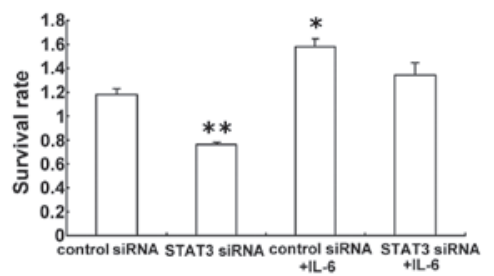

$\mathbf{F}$

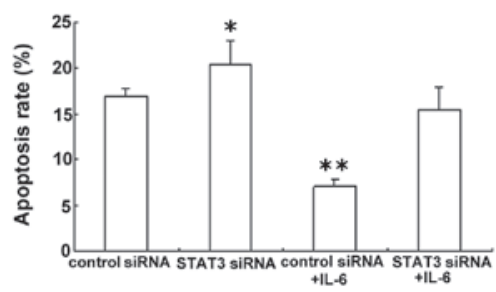

Figure 4. Downregulation of JAK/STAT3 expression inhibits proliferation stimulated by IL-6 in PMVECs. (A) JAK2 was knocked down in the PMVECs. (B) Cell survival was analyzed using MTT following JAK2 knock down. (C) Apoptosis was detected by flow cytometry in the cells with JAK2 knock down. (D) STAT3 was knocked down in the PMVECs. (E) Cell proliferation was assayed by MTT method in the cells with STAT3 knock down. (F) Apoptosis was detected by flow cytometry in the cells with STAT3 knock down. The results are representative of three independent experiments with similar results. " $\mathrm{P}<0.05,{ }^{* * *} \mathrm{P}<0.01$ vs. control siRNA. JAK, janus kinase; STAT, signal transducer and activator of transcription; IL, interleukin PMVECs, pulmonary microvascular endothelial cells; p-, phosphorylated; si, small interfering.

according to the manufacturer's instructions (BD Biosciences, San Jose, CA, USA). The apoptosis rate (\%) of the stained cells was analyzed using a Beckman Coulter Epics Altra II cytometer (Beckman Coulter, Brea, CA, USA).
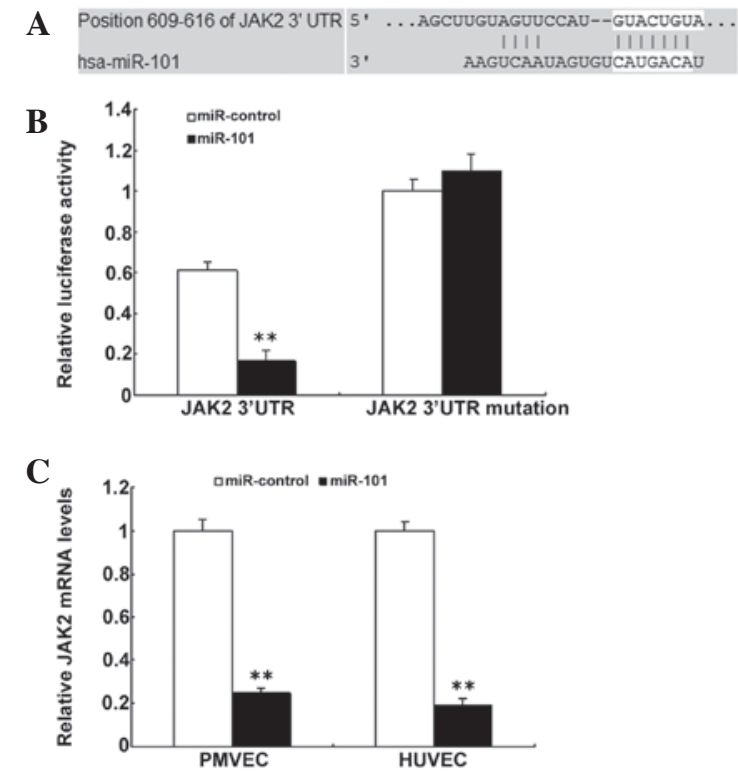

D

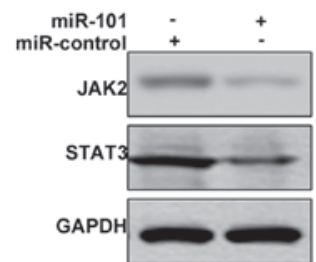

Figure 5. miR-101 downregulates JAK2 expression directly. (A) The 3'-UTR of the JAK2 gene contains binding sites for miR-101 according to bioinformatic analysis. (B) miR-101 suppressed the expression of a luciferase reporter gene harbouring the 3'-UTR of JAK2. Data are presented as relative firefly luciferase activity normalized to Renilla luciferase activity from the same construct. The data presented are presented as the mean \pm standard deviation collected from three independent experiments. miR-101 restoration downregulated JAK2 (C)mRNA and (D) protein in PMVECs. Cells were transfected with miR-101 or miR-control (100 pmol per well in 6-well plates by Lipofectamine 2000) for $48 \mathrm{~h}$, then collected for reverse transcription-quantitative polymerase chain reaction and western blot analysis, respectively. ${ }^{* *} \mathrm{P}<0.01$ vs. miR-control. miR, microRNA; JAK, janus kinase; UTR, untranslated region; PMVECs, pulmonary microvascular endothelial cells; STAT, signal transducer and activator of transcription.

Statistical analysis. Data were analyzed using SPSS 13.0 software (SPSS, Inc., Chicago, IL, USA) and presented as the mean \pm standard error of the mean \pm standard error of the mean of at least three independent experiments. Two-tailed Student's t-test was used for comparisons of two independent groups. Gene expression was analyzed by a Mann-Whitney U test. $\mathrm{P}<0.05$ was considered to indicate a statistically significant difference.

\section{Results}

IL-6 expression in rat models of HPS. To investigate whether cytokine production is induced in HPS, rat models were established. The cytokine profile was assayed by a microarray analysis. The data showed that TNF- $\alpha$, IL-8, IL-1 and IL-6 mRNA were increased compared with the controls. This result was confirmed by RT-qPCR (Fig. 1A). Notably, IL-6 mRNA levels were significantly increased compared with TNF- $\alpha$, IL-8 and IL-1. Next, IL-6 protein from the cultured lung tissue was analyzed ELISA (Fig. 1B). The 

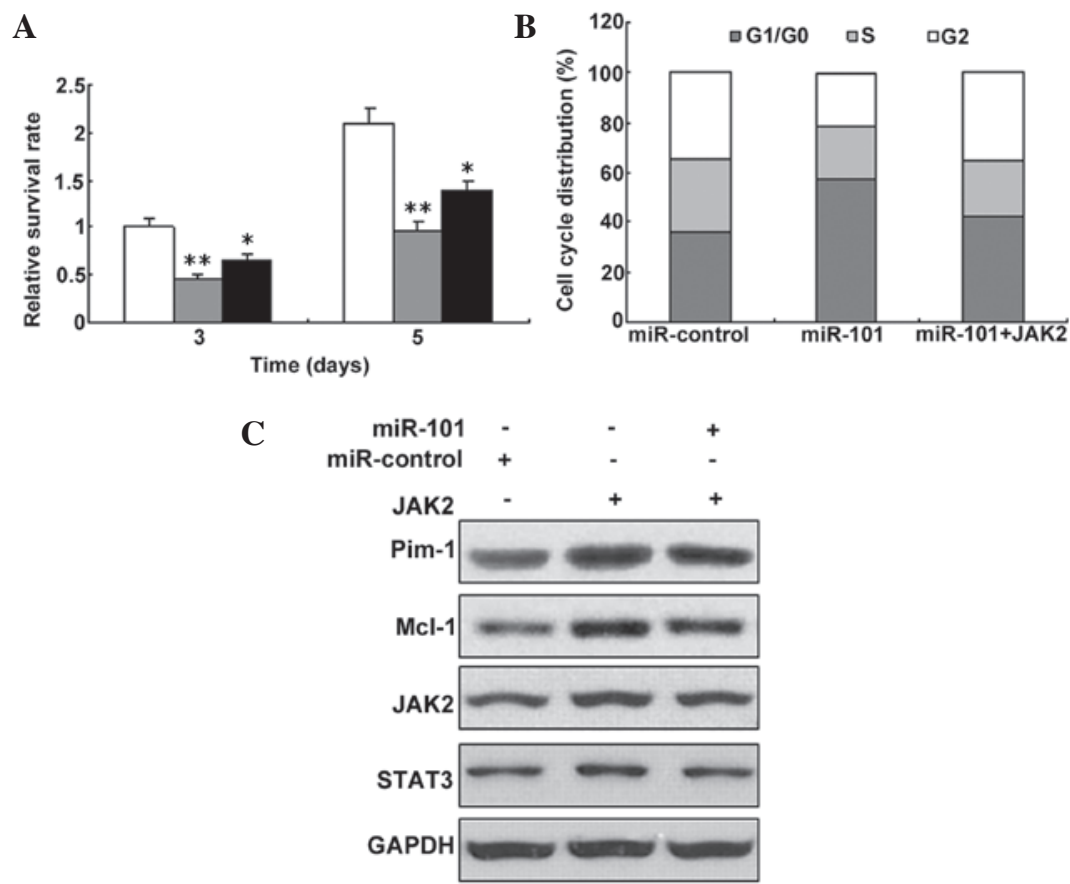

Figure 6. miR-101 inhibits proliferation of PMVECs targeting JAK2. (A) miR-101 inhibited proliferation of PMVECs with JAK2 overexpression as observed by an MTT assay. (B) miR-101 induced changes in cell cycle distribution as determined by flow cytometry. (C) miR-101 inhibited the JAK2/STAT3 signal pathway in the cells as observed by western blot analysis. ${ }^{*} \mathrm{P}<0.05,{ }^{* *} \mathrm{P}<0.01$ vs. control. miR, microRNA; PMVECs, pulmonary microvascular endothelial cells; JAK, janus kinase; STAT, signal transducer and activator of transcription.

result indicated that IL-6 protein was also increased in the rat models.

IL-6 promotes proliferation and inhibits LPS-induced apoptosis in PMVECs. Based on the above data, PMVECs were used to investigate the role of IL-6. PMVEC proliferation was analyzed by the MTT method. The data showed that IL-6 promoted cell proliferation (Fig. 2A). LPS can induce cell apoptosis; thus, in order to determine whether IL-6 protects cells from apoptosis, PWVECs were treated with LPS, with or without IL-6, and cell apoptosis was analyzed by flow cytometry. It was determined that IL-6 decreased cell apoptosis induced by LPS (Fig. 2B and C).

IL-6 activates the JAK/STAT3 signaling pathway in pulmonary microvascular endothelial cells. IL-6 is induced during HPS. To examine the possibility that secretory factors from PMVECs could activate the JAK/STAT3 signaling pathway, the cells were treated with IL- 6 for $12 \mathrm{~h}$, and phosphorylation of JAKs was then monitored by western blot analysis. As shown in Fig. 3A, phosphorylation of JAK2 was increased significantly, when the cells were treated with IL-6 for $12 \mathrm{~h}$, but not JAK1 and JAK3 phosphorylation. mRNA levels of JAK1, JAK2 and JAK3 were not changed, but STAT3 mRNA levels increased greatly in the cells following IL-6 treatment. (Fig. 3B).

IL-6-induced downregulation of JAK expression inhibits proliferation and induces apoptosis in PMVECs. To investigate the role of JAK2/STAT3 in IL-6-induced cell proliferation, an MTT assay was performed on PMVECs with JAK2 downregulation to analyze cell proliferation. The data showed that
JAK2 was knocked down effectively by RNAi and STAT3 was inactive (Fig. 4A). Downregulation of JAK2 could inhibit cell proliferation of PWVECs exposed to IL-6 (Fig. 4B). After JAK2 down-regulation, the cell apoptosis rate increased, but when the cells were treated with IL-6, the apoptosis rate began to decrease (Fig. 4C). To further investigate whether STAT3 has a role in PMVECs, STAT3 was knocked down and not active in the cells with STAT3 siRNA (Fig. 4D). STAT3 was observed to promote PMVEC cell proliferation and inhibit apoptosis (Fig. 4E and F).

$J A K 2$ is a target gene of miR-101. Bioinformatic prediction indicated that JAK2 may be a target gene of miR-101 (Fig. 5A). The pGL3 plasmid was modified by adding the human 3'-UTR or the 3'-UTR with mutations in regions complementary to miR-101 seed regions downstream of the firefly luciferase gene. PMVECs were transiently co-transfected with negative control (mock) or miR-101 together with the indicated luciferase constructs, and luciferase activity was analyzed after $48 \mathrm{~h}$. When the PMVECs were co-transfected with miR-101 or luciferase vectors and pGL3-JAK2-Mut or pGL3-JAK2WT, the luciferase activity was markedly lower in cells with pGL3-JAK2-WT transfection and was rescued in the cells with pGL3-JAK2-Mut transfection (Fig. 5B). JAK2 mRNA was decreased in the PMVECs with miR-101 treatment than the control (Fig. 5C). JAK2 protein was also decreased in PMVECs with miR-101 treatment compared with the control (Fig. 5D).

miR-101 inhibits pPMVEC proliferation by targeting the IL-6/JAK2/STAT3 pathway. Given the fact that JAK2 was the target gene of miR-101, the present study aimed to determine 
whether miR-101 regulates the proliferation of PMVECs. It was tested whether miR-101 inhibits the proliferation of the cells with overexpression of JAK2. The result showed that miR-101 in PMVECs inhibited cell proliferation with JAK2 overexpression (Fig. 6A). Since the JAK/STAT3 signaling pathway is an important pathway involved in PMVECs, the levels of several proteins and downstream proteins in the JAK/STAT3 signaling pathway were analyzed. To further investigate cell proliferation, the cell cycle was analyzed by flow cytometry, and the results showed that miR-101 could induce G1 phase arrest (Fig. 6B). A downstream protein of the JAK/STAT3 singling pathway, Pim-1, was decreased by miR-101 over-expression. A similar result was obtained for Mcl-1, an important downstream protein of this pathway (Fig. 6C).

\section{Discussion}

In the present study, the mechanism of proliferation of PMVECs in HPS was investigated. It was determined that IL-6 was enhanced in rat models of HPS and promoted cell proliferation and inhibited apoptosis. In addition, cell proliferation was inhibited and apoptosis was induced in the cells with JAK2 or STAT3 downregulation. Thus a novel role of miR-101 in regulating cell proliferation by suppressing JAK2 expression in PMVECs was determined.

IL-6 is a pro-inflammatory cytokine characterized as a potent activator of STAT3. They function cooperatively to promote cellular proliferation and inhibit apoptosis (14). As shown in Fig. 1, a significant difference in the expression of IL-6 was observed between HPS and normal rats. This study focused on the IL-6/JAK/STAT3 signaling pathway and investigated its function in PMVECs. As expected, the presence of high IL-6 and p-STAT3 expression was observed in PMVECs, and a significant correlation was observed between the two. This indicated the activation of JAK/STAT3 signaling in HPS. Similar results were obtained by Jiang et al (15), where it was reported that IL-6 promoted STAT3 activation significantly at the posttranslational level in vitro and indicated that IL-6/STAT3 signaling was involved in human biliary epithelial cell migration and wound healing.

Binding of IL- 6 to the IL-6 receptor can phosphorylate the tyrosine of gp130, and further activate JAK family members including JAK1, JAK2, and tyrosine kinase2 (Tyk2). STAT3 is phosphorylated by activated JAK and translocates into the nucleus to control the expression of substrates (14). STAT3 mediates signal transduction and transcription via various cytokines and growth factors. p-STAT3 accelerates the cell cycle, promotes cellular differentiation and inhibits apoptosis through regulating the expression of Cyclin D1, C-myc, Bcl-xl and vascular endothelial growth factor (VEGF), which is an essential factor in cell proliferation (16). The present results are consistent with previous results and propose that the IL-6/JAK/ STAT3 signaling pathway is active in PMVECs and may represent a novel therapeutic target. In recent years, inhibitors of the IL-6/JAK/STAT3 signaling pathway have emerged as a promising treatment option for certain diseases. For instance, siRNA-STAT3 therapy was demonstrated to be effective in ovarian cancer, and a suppressor of cytokine signaling treatment was also demonstrated to be active in blocking the STAT3 signaling in liver disease such as cirrhosis (17-21). This study has provided insight into a potential strategy for the treatment of tumors and other proliferative diseases, such as HPS.

There are certain studies that demonstrated that miR-101 is important in cancer cell proliferation by targeting ZEB1, ZEB2, Rac1, RanBP9, Cox-2, EZH2, CPEB1 and Stathmin1 (22-28). However, in HPS, there has not previously been a study to determine the role of miRNA-101. The present study identified that JAK2 was regulated by miR-101 in PMVECs, and that miR-101 inhibited cell proliferation.

In conclusion, the present study demonstrated that miR-101 inhibited the cell proliferation of PMVECs by downregulation of JAK2. Low miR-101 expression may be an unfavorable prognostic factor in patients with HPS. The data in the present study indicates that miR-101 may be a therapeutic target in HPS. In addition, the present findings expand the knowledge of the pathogenic mechanisms underlying HPS.

\section{References}

1. Eshraghian A, Kamyab AA and Yoon SK: Pharmacological treatment for hepatopulmonary syndrome. Biomed Res Int 2013: 670139, 2013.

2. Polavarapu $\mathrm{N}$ and Tripathi D: Liver in cardiopulmonary disease. Best Pract Res Clin Gastroenterol 27: 497-512, 2013.

3. Grace JA and Angus PW: Hepatopulmonary syndrome: Update on recent advances in pathophysiology, investigation and treatment. J Gastroenterol Hepatol 28: 213-219, 2013.

4. Zhang $J$ and Fallon MB: Hepatopulmonary syndrome: Update on pathogenesis and clinical features. Nat Rev Gastroenterol Hepatol 9: 539-549, 2012.

5. Sussman NL, Kochar R and Fallon MB: Pulmonary complications in cirrhosis. Curr Opin Organ Transplant 16: 281-288, 2011.

6. Zhang ZJ and Yang CQ: Progress in investigating the pathogenesis of hepatopulmonary syndrome. Hepatobiliary Pancreat Dis Int 9: 355-360, 2010.

7. Valenti A and Caimi G: Physiopathological, clinical and therapeutic aspects of hepatopulmonary syndrome. Clin Ter 161: e123-e128, 2010

8. Macêdo LG and Lopes EP: Hepatopulmonary syndrome: An update. Sao Paulo Med J 127: 223-230, 2009.

9. Ho V: Current concepts in the management of hepatopulmonary syndrome. Vasc Health Risk Manag 4: 1035-1041, 2008.

10. Rodríguez-Roisin $\mathrm{R}$ and Krowka MJ: Hepatopulmonary syndrome-a liver-induced lung vascular disorder. N Engl J Med 358: 2378-2387, 2008.

11. Varghese J, Ilias-basha H, Dhanasekaran R, Singh S and Venkataraman J: Hepatopulmonary syndrome-past to present. Ann Hepatol 6: 135-142, 2007.

12. Kartha RV and Subramanian S: Competing endogenous RNAs (ceRNAs): New entrants to the intricacies of gene regulation. Front Genet 5: 8, 2014.

13. Banno K, Iida M, Yanokura M, Kisu I, Iwata T, Tominaga E, Tanaka K and Aoki D: MicroRNA in cervical cancer: OncomiRs and tumor suppressor miRs in diagnosis and treatment. ScientificWorldJournal 2014: 178075, 2014.

14. Chang Q, Bournzou E, Sansone P, Berishaj M, Gao SP, Daly L, Wels J, Theilen T, Granitto S, Zhang X, et al: The IL-6/JAK/STAT3 feed-forward loop drives tumorigenesis and metastasis. Neoplasia 15: 848-862, 2013.

15. Jiang GX, Zhong XY, Cui YF, et al: IL-6/STAT3/TFF3 signaling regulates human biliary epithelial cell migration and wound healing in vitro. Mol Biol Rep 37: 3813-3818, 2010.

16. Wang G, Qian P, Jackson FR, Qian G and Wu G: Sequential activation of JAKs, STATs and xanthine dehydrogenase/oxidase by hypoxia in lung microvascular endothelial cells. Int J Biochem Cell Biol 40: 461-470, 2008.

17. Xiong H, Zhang ZG, Tian XQ, Sun DF, Liang QC, Zhang YJ, Lu R, Chen YX and Fang JY: Inhibition of JAK1, 2/STAT3 signaling induces apoptosis, cell cycle arrest and reduces tumor cell invasion in colorectal cancer cells. Neoplasia 10: 287-297, 2008. 
18. Januma N, Shima H, Nakamura K and Kikuchi K: Protein tyrosine phosphatase epsilonC selectively inhibits interleukin-6- and IL-10- induced JAK-STAT signaling. Blood 98: 3030-3034, 2001.

19. Takeda K and Akim S: Stat family of transcription factors in cytokine-mediated biological responses. Cytokine Growth Factor Rev 11: 199-207, 2000.

20. Kuczkowski J, Sakowicz-Burkiewica M, Izycka-Swieszewska E, Mikaszewski B and Pawełczyk T: Expression of tumor necrosis factor- $\alpha$, interleukin- $1 \alpha$, interleukin- 6 and interleukin-10 in chronic otitis media with bone osteolysis. ORL J Otorhinolaryngol Relat Spec 73: 93-99, 2011.

21. Nason R, Jung JY and Chole RA: Lipopolysaccharide-induced osteoclastogenesis from mononuclear precursors: A mechanism for osteolysis in chronic otitis. J Assoc Res Otolaryngol 10 151-160, 2009.

22. Guo F, Cogdell D, Hu L, Yang D, Sood AK, Xue F, and Zhang W: miR-101 suppresses the epithelial-to-mesenchymal transition by targeting ZEB1 and ZEB2 in ovarian carcinoma. Oncol Rep 31: 2021-2028, 2014

23. Lin X, Guan H, Li H, Liu L, Liu J, Wei G and Zhang W: miR-101 inhibits cell proliferation by targeting Racl in papillary thyroid carcinoma. Biomed Rep 2: 122-126, 2014.
24. Barbato C, Pezzola S, Caggiano C, Antonelli M, Frisone P, Ciotti MT and Ruberti F: A lentiviral sponge for miR-101 regulates RanBP9 expression and amyloid precursor protein metabolism in hippocampal neurons. Front Cell Neurosci 8: 37, 2014.

25. Lin C, Huang F, Zhang YJ, Tuokan T and Kuerban G: Roles of MiR-101 and its target gene Cox-2 in early diagnosis of cervical cancer in Uygur women. Asian Pac J Cancer Prev 15: 45-48, 2014.

26. Wang Y, Xiang W, Wang M, Huang T, Xiao X, Wang L, Tao D, Dong L, Zeng F and Jiang G: Methyl jasmonate sensitizes human bladder cancer cells to gambogic acid-induced apoptosis through down-regulation of EZH2 expression by miR-101. Br J Pharmacol 171: 618-635, 2014.

27. Xiaoping L, Zhibin Y, Wenjuan L, Zeyou W, Gang X, Zhaohui L, Ying Z, Minghua W and Guiyuan L: CPEB1, a histone-modified hypomethylated gene, is regulated by miR-101 and involved in cell senescence in glioma. Cell Death Dis 4: e675, 2013.

28. Wang R, Wang HB, Hao CJ, Cui Y, Han XC, Hu Y, Li FF, Xia HF and Ma X: MiR-101 is involved in human breast carcinogenesis by targeting Stathmin1. PLoS One 7: e46173, 2012. 